\title{
Síndrome de Binder. ¿Cuál es el tipo idóneo de cirugía?
}

\section{Binder syndrome. What type of surgery is ideal?}

\author{
Lorena Pingarrón Martín*, Teresa González Otero, Ignacio Navarro Cuéllar, \\ Natalia Gisbert Alemany y Miguel Burgueño García
}

Servicio de Cirugía Oral y Maxilofacial, Hospital Universitario La Paz, Madrid, España

\section{Síndrome de Binder}

El síndrome de Binder o displasia maxilonasal es una malformación congénita descrita por Allan Ragnell en 1952 con la denominación de anomalía en «cara de plato» y 10 años después por Binder, quien le otorgó nombre propio al describir detalladamente esta entidad ${ }^{1,2}$. Desde entonces alrededor de 250 casos se han recogido en la literatura.

Fisiopatológicamente, se trata de una deformidad estructural con inhibición del crecimiento óseo, en especial en el área subnasal donde aparecen pequeñas excavaciones a cada lado de la espina nasal, la cual se encuentra retruida.

La inhibición de los centros de osificación en los bordes laterales e inferiores de la apertura piriforme, entre la quinta y sexta semana de desarrollo gestacional, dará lugar a una hipoplasia localizada del maxilar con la consiguiente facies típica del paciente afecto de este síndrome, con retracción de la unión labio columelar y anormalidad en la forma de la base columelar, similar a la que presentan los pacientes con labio leporino bilateral ${ }^{3}$.

La etiología no está clara, se considera multifactorial fruto de una mezcla de factores genéticos y medioambientales. Pese a que la mayor parte de los casos son esporádicos, en el 16\% de los pacientes con síndrome de Binder se admite un patrón de herencia autosómica recesiva con penetrancia incompleta ${ }^{4}$.

Clínicamente la típica apariencia de esta entidad es un ángulo frontonasal de casi 180 grados, columela corta, labio superior convexo e hipoplasia de la premaxila.
Binder, en su descripción original, enumeró seis características fenotípicas: ausencia de ángulo frontonasal, posición anormal de los huesos propios nasales, hipoplasia intermaxilar con la consecuente maloclusión, reducción o ausencia de la espina nasal anterior, atrofia de la mucosa nasal y ausencia de seno frontal (esta última característica no es obligatoria). Todos los pacientes con síndrome de Binder presentan estas características en algún grado de afectación.

Una adecuada estrategia terapéutica requiere contestar a dos consideraciones fundamentales: 1) ¿cuál es la técnica quirúrgica apropiada? y 2) ¿cuál es la edad óptima a la que se debe intervenir?

\section{¿Cuál es el tipo idóneo de cirugía?}

Dado que el grado de malformación en el síndrome de Binder varía significativamente, el planteamiento quirúrgico necesita individualizarse. Antes de comenzar ningún tratamiento, los pacientes deben ser evaluados por todos los especialistas implicados para establecer un adecuado plan de corrección. El método óptimo para manejar estos casos comienza, si el caso lo requiere, con el tratamiento ortodóncico de la maloclusión seguido de cirugía ortognática. La corrección nasal y del tercio medio vendrá a continuación.

Autores como Holmström y Gewalli, en su análisis antropométrico digitalizado realizado en 92 pacientes sometidos a diferentes técnicas reconstructivas a lo largo de 23 años ${ }^{5}$, concluyen que la incisión bicoronal o el abordaje septo-columelar

Véase contenido relacionado en DOI:

10.1016/j.maxilo.2010.12.002.

* Autor para correspondencia.

Correo electrónico: lorenapingarron@yahoo.es (L. Pingarrón Martín). 
no son opciones adecuadas para acceder al foco de la malformación en torno a la apertura piriforme. Afirman que el abordaje intraoral es el mejor acceso para la liberación de los tejidos blandos circunscritos y minimizar así las fuerzas aplicadas a los injertos colocados. Dado que el foco de reconstrucción debe centrarse en el área local de hipoplasia subnasal, la osteotomía de Le Fort II no corregiría este defecto patognomónico o la escasez de proyección de la punta. La técnica de Le Fort I descrita por Posnick y Tompson ${ }^{6}$ debería reservarse para los pacientes que asocian una anomalía dentoesquelética clase III, lo cual se produce en el 54\% de los casos, y estaría indicado realizar la corrección maxilomandibular antes de la reconstrucción nasal ${ }^{5}$.

Los injertos óseos y cartilaginosos han sido utilizados tradicionalmente como tratamiento reconstructivo de la hipoplasia maxilonasal.

Ragnell describió por primera vez la utilización de chips de hueso esponjoso de cresta iliaca para la superficie anterior maxilar, a través de una incisión medial en la base columelar ${ }^{1}$. Para mejorar el contorno nasal Holmström, Losken y Rune utilizaron la técnica de injertos óseos en «L» utilizando como zona donante la cresta iliaca y la calota. Estos autores utilizaron a su vez chips óseos injertados en la premaxila a través de un abordaje intraoral vestibular o a partir del pliegue nasal perialar.

Sin embargo, los resultados con injerto óseo continúan siendo impredecibles, con frecuente reabsorción del mismo, en especial si el tejido blando que lo recubre es fino, así como malos resultados a largo plazo en caso de desplazamiento del hueso injertado. Uno de los principales problemas de esta técnica es que los pacientes se quejan a menudo de excesiva rigidez de la punta nasal.

El injerto de cartílago en «L» ha sido abandonado dada la evolución impredecible y el escaso remodelado positivo.

Por otro lado, el injerto de cartílago costal mantiene su volumen a largo plazo y proporciona a la nariz una apariencia más natural, designándolo como el injerto de elección para el aumento del dorso. Para prevenir la torsión y doblez de los largos injertos requeridos para el dorso, deben obtenerse de la parte central de la costilla y sumergirlos en solución de cloruro sódico al 0,9\% durante 30 minutos.

Un concepto de suma importancia es la deficiencia de tejidos blandos en la columela de estos pacientes, causante fundamental de la nariz alada del síndrome de Binder. Para solucionar esto, Holmström y Gewalli ${ }^{5}$ proponen el alargamiento de la columela con plastia en V-Y si lo que existe es un verdadero acortamiento de la piel, pero si lo que se presenta es únicamente una retracción por la hipoplasia del suelo nasal, el avance de la piel puede conseguirse a partir de la liberación de la piel labio-columelar con la ayuda de strut cartilaginoso para mantener la adecuada proyección.

Estos mismos autores ${ }^{5}$ aconsejan la reposición anterior del septo nasal cuando la pirámide ósea posee altura adecuada, explicando la adecuada permanencia de la proyección de la punta nasal por la liberación de los tejidos blandos realizada previamente al injerto.

Recientemente, los implantes porosos de alta densidad de polietileno se han descrito como una buenas alternativa para pacientes con síndrome de Binder que no aceptan la reconstrucción con injerto costal y/o cirugía ortognática ${ }^{7}$.

\section{¿Cuál es el momento idóneo de la cirugía?}

Cirujanos craneofaciales de París y Ciudad de Méjico (D. Marchac y F. Ortiz-Monasterio) abogan por la cirugía temprana a los 7-10 años de edad ${ }^{8}$. Una segunda intervención en la adolescencia tardía sería necesaria en este caso. Para conseguir la mejor integración social del paciente, algunos autores recomiendan realizar la cirugía antes de comenzar la etapa escolar. La técnica de injerto onlay parece influenciar positivamente al crecimiento facial con posibilidad de correcciones secundarias menores en cualquier etapa del desarrollo ${ }^{9}$.

En contra, Tessier defendió el adecuado tratamiento quirúrgico de estos pacientes a partir de los 16 años, una vez completado el desarrollo esquelético del individuo.

En Suecia, siguen esta misma tendencia, siendo partidarios de posponer la cirugía hasta completar el desarrollo del paciente, como Holmström y Gewalli ${ }^{5}$ quienes defienden un único tiempo reconstructivo, con excepción de casos severos en los que estaría indicado un primer tiempo quirúrgico para reparar la deformidad dentofacial y un segundo tiempo para tratar la malformación nasal.

\section{B I B L I O G R A F Í A}

1. Ragnell A. A simple method of reconstruction in some cases of dish-face deformity. Plast Reconstr Surg. 1952;10:227.

2. Binder KH. Dysostosis maxillo-nasalis, ein arhinen-cephaler Missbildungkomplex. Dtsch Zahnaerztl Z. 1962:438.

3. Draf W, Bockmühl U, Hoffmann B. Nasal correction in maxillonasal dysplasia (Binder's syndrome): a long term follow-up study. Br J Plast Surg. 2003;56:199-204.

4. Holmström H. Clinical and pathologic features of maxillonasal dysplasia (Binder's syndrome): Significance of the prenatal fossa on etiology. Plast Reconstr Surg. 1986;78:559.

5. Holmström H, Gewalli F. Long-Term Behavior of Three Different Grafts in Nasomaxillary Reconstruction of Binder Syndrome: An Analysis by Digitalized Measurements. Plast Reconstr Surg. 2008;122:1524-34.

6. Posnick JC, Tompson B. Binder syndrome: Staging of reconstruction and skeletal stability and relapse patterns after LeFort osteotomy using miniplate fixation. Plast Reconstr Surg. 1997;99:961.

7. Seyhan T, Kircelli BH, Caglar B. Correction of Septal and Midface Hypoplasia in Maxillonasal Dysplasia (Binder's Syndrome) Using High-Density Porous Polyethylene. Aesthetic Plast Surg. 2009.

8. Monasterio FO, Molina F, McClintock JS. Nasal correction in Binder's syndrome: the evolution of a treatment plan. Aesthetic Plast Surg. 1997;21:299-308.

9. Rune B, Aberg M. Bone grafts to the nose in Binder's syndrome (maxillonasal dysplasia): A follow-up of eleven patients with the use of profile roentgenograms. Plast Reconstr Surg. 1998;101:297.

doi:10.1016/j.maxilo.2011.06.001 